\title{
Fault-Section Location of Distribution Network based on Adaptive Mutation Shuffled Frog Leaping Algorithm
}

\author{
Yanzhou Sun, Han Wu*, Yawei Zhu, Yanfang Wei, and Tieying Zhao \\ School of Electrical Engineering and Automation, Henan Polytechnic University, Jiaozuo, 454000, China
}

\begin{abstract}
Fast and accurate identification of feeder fault section plays a crucial role in improving the stability of distribution networks. We address the low accuracy and unsatisfactory effect of traditional algorithms when the fault indicator has been used for fault location of distribution network lines. This paper proposes a kind of fault location method based on an adaptive mutation shuffled frog leaping algorithm (AMSFLA), which introduces an adaptive mutation factor. This proposed method was validated by simulation with a typical IEEE 33-bus distribution network model and has been shown to effectively solve the premature problem that exists in the classical shuffled frog leaping algorithm (SLFA), as well as speed up the calculation and accurately locate the fault section when multiple point faults and fault signal distortion occur.
\end{abstract}

Keywords: distribution network; fault indicator; shuffled frog leaping algorithm (SLFA); adaptive mutation factor; fault-section location

(Submitted on December 23, 2018; Revised on February 10, 2019; Accepted on April 15, 2019)

(C) 2019 Totem Publisher, Inc. All rights reserved.

\section{Introduction}

With the rapid development of the economy and an increasing demand of electricity, grids tend to be more complicated. The power supply service continuity is one of the most important concerns of utility companies. When the distribution network has faults, it is of great significance to identify the fault effectively and locate the fault section accurately to ensure a stable distribution network and reduce economic loss [1-2]. The current methods include the "S" injection method [3], zero sequence current method [4], impedance method [5], fault indicator method [6], etc. Compared to the first three methods, the fault location method based on fault indicator (FI) has been widely used because of its low cost and ability to find fault points easily [7-9].

The principle of the fault indicator location method is to install the FI in sections of the distribution line, and use it to monitor the line condition in real time. When the fault current is detected, the FI status will be transferred to the main control center for processing, and then the fault section location is achieved. The focus of FI is on accurate, fast and faulttolerant algorithms [10-11]. The matrix algorithm has the advantages of simplicity and high speed, but the reliability of the algorithm will be reduced when the information of the distribution network is distorted [12-13]. Artificial intelligence algorithms can achieve satisfactory results, such as genetic algorithm, immune algorithm, ant colony algorithm, etc., but these algorithms have disadvantages like low efficiency, slow convergence speed, easily causing local convergence, and low general fault location efficiency [14-15].

In order to increase the location efficiency of traditional intelligent algorithms and solve the problems of convergence and local optimization frequently, a fault location algorithm based on an adaptive mutation shuffled frog leaping algorithm (AMSFLA) is proposed. The simulation results show that the algorithm is effective and has achieved the purpose of locating fault sections quickly and accurately in distribution networks.

\footnotetext{
* Corresponding author.

E-mail address: hpuwwwh@163.com
} 


\section{Modified Shuffled Frog Leaping Algorithm}

SFLA is a new intelligent algorithm based on a social group cooperation proposed by M. M. Eusuff and K. E. Lansey in 2003 [16]. By simulating the foraging behavior of frog populations, combined with the advantages of the genetic algorithm and particle swarm optimization algorithm, the new algorithm has a simple mathematical model and easy-to-solve multiobjective optimization problem [17-18].

\subsection{Basic Principles of SFLA}

SFLA is an optimal solution algorithm for simulating information exchange when frogs are foraging in restricted ponds. Firstly, the initial frog population is generated and ranked according to the fitness value, and the optimal individual is recorded. Secondly, the frogs are taken into different groups and the worst and the best individuals are recorded. Thirdly, a local search uses the frog leap update formula to update the worst. If the updated individual is better, the original worst will be replaced; otherwise, it will be replaced by the optimal individual of the whole frog population. Finally, after updating the times of local iterations, the frog group will be re-mixed, sorted and grouped again until the global iterations are reached and the algorithm stops.

The process of SFLA compares four steps, namely, the initial population structure, the sub-group division, the local search strategy and the global information exchange. According to the following, a detailed analysis has been given for each part:

\subsubsection{The Initial Population Structure}

With $P$ frogs $\left(X_{1}, X_{2}, \cdots, X_{P}\right)$, as the initial population, the frog $i$ in the group represents the solution $i$, which is expressed by $X_{i}=\left(x_{i 1}, x_{i 2}, \cdots, x_{i Q}\right)$ with $Q$ as the dimension of the solution.

\subsubsection{The Sub-Group Division}

Firstly, the frogs in the group are ranked according to fitness value, and the individual with the global best fitness value is recorded as $X_{z}$. Then, the frog group is divided into $m$ sub-groups, and the number of frogs in each sub-group is $n$. For example, the first frog is placed in group 1, the second frog in group 2, the $m$ frog in group $m$, and the frog in group 1, and so on until the distribution is completed. In each group, based on fitness value, the best is recorded as $X_{b}$ and the worst is recorded as $X_{w}$.

\subsubsection{The Local Search Strategy}

Local search is the most crucial step in SFLA. When local search is carried out, the individual with the worst fitness $X_{w}$ in the sub-group is updated. The updated strategy is as follows:

$$
\begin{gathered}
D_{i}=\operatorname{rand}() \cdot\left(X_{b}-X_{w}\right) \\
X_{w}^{\prime}=X_{w}+D_{i}
\end{gathered}
$$

Where $i$ represents the frog $i$ in the sub-group, $i=1,2, \cdots, n$ and $D_{i}$ is the updating step length of the frog, $-D_{\max } \leq D_{i} \leq D_{\max } \cdot \operatorname{rand}()$ is a random number between 0 and $1 . D_{\max }$ is the maximum step length that the frog is allowed to move. If the updated position is better than before, the solution is replaced by the updated solution, otherwise it replaces the sub-group best $X_{b}$ in Equation (1) with the global best $X_{z}$. If the updated position is still not as good as the $X_{w}$, it randomly generates a solution to replace the worst. It repeats the above operations within the specified times of internal iterations to complete the local search.

\subsubsection{The Global Information Exchange}

When the internal updating of the sub-group is completed, all sub-groups are merged into a new group, ranked according to 
fitness. The sub-group division and the local search strategy of the sub-group are re-executed. Repeat this until it outputs the best solution

\subsection{Adaptive Mutation Shuffled Frog Leaping Algorithm}

The SFLA has advantages like simple principle, few parameters and easily finding the optimal solution, but it also has some shortcomings, such as uneven initial population, poor adaptability of moving step, slow search speed and easily falling into local optimization [19-20]. In order to solve the problems mentioned above, the SFLA is improved by introducing adaptive local search factor and levy mutation factor into local search strategy, which significantly increases the search speed and performance of this algorithm.

\subsubsection{Adaptive Local Search Factor}

Because of the complexity of the distribution network, the SFLA often falls into local optimization and cannot find all the fault in the distribution network in time. This paper proposes an adaptive local search factor $\omega$, which is introduced into the local search strategy of the algorithm to balance global and local search capabilities so that the algorithm can quickly find all the fault points in the distribution network and achieve accurate fault location. Therefore, Equation (1) becomes Equation (3):

$$
D_{i}=\omega D_{i}^{\prime}+\operatorname{rand}() \cdot\left(X_{b}-X_{w}\right)
$$

Where $\omega$ is the inertia weight and $D_{i}^{\prime}$ is the moving step size of the previous worst frog. Adding inertia weight $\omega$ here avoids falling into precocity when the local best solution is near, and fast search improves the convergence speed when it is located near the global best. As such, the linear descending adjustment is $\omega$. $\omega$ should be a larger value at first, which is conducive to global search. When approaching the best, the value of $\omega$ is reduced, which is useful for careful search, and the value of $\omega$ can be adjusted according to Equation (4).

$$
\omega=\omega_{\min }+\left(\omega_{\max }-\omega_{\min }\right) \times \frac{T-t}{T}
$$

\subsubsection{Levy Mutation Factor}

In order to ensure the accuracy of fault location in distribution networks, the levy mutation factor based on levy distribution has been introduced into this algorithm. Compared with the Gauss mutation operator and Cauchy mutation operator, the levy mutation factor has a better mutation step and can produce stronger disturbance. The levy mutation factor enhances the global optimization ability of the algorithm in a complex distribution network and achieves accurate fault location when the fault signal is distorted. We propose to add a levy mutation operator $L_{j}(t)$ to Equation (3), which then becomes Equation (5):

$$
D_{i}=\left[\omega D_{i}^{\prime}+\operatorname{rand}() \cdot\left(X_{b}-X_{w}\right)\right] \times L_{j}(t)
$$

Where $L_{j}(t)$ is a random function that obeys the Levy distribution.

After adding the adaptive mutation factor in the algorithm, the updating equations of the new local update strategy are as follows:

$$
\begin{gathered}
D_{i}=\left[\omega D_{i}^{\prime}+\operatorname{rand}() \cdot\left(X_{b}-X_{w}^{\prime}\right)\right] \times L_{j}(t) \\
X_{w}^{\prime}=X_{w}+D_{i}
\end{gathered}
$$

\subsection{The Flow of Adaptive Mutation Shuffled Frog Leaping Algorithm}

After introducing the adaptive mutation operator into the SFLA, the coding and grouping of the algorithm are not affected. As such, only the local search strategy is modified, which greatly improves the iteration speed and global search accuracy of the traditional algorithm. The flow chart of AMSFLA is shown in Figure 1. 


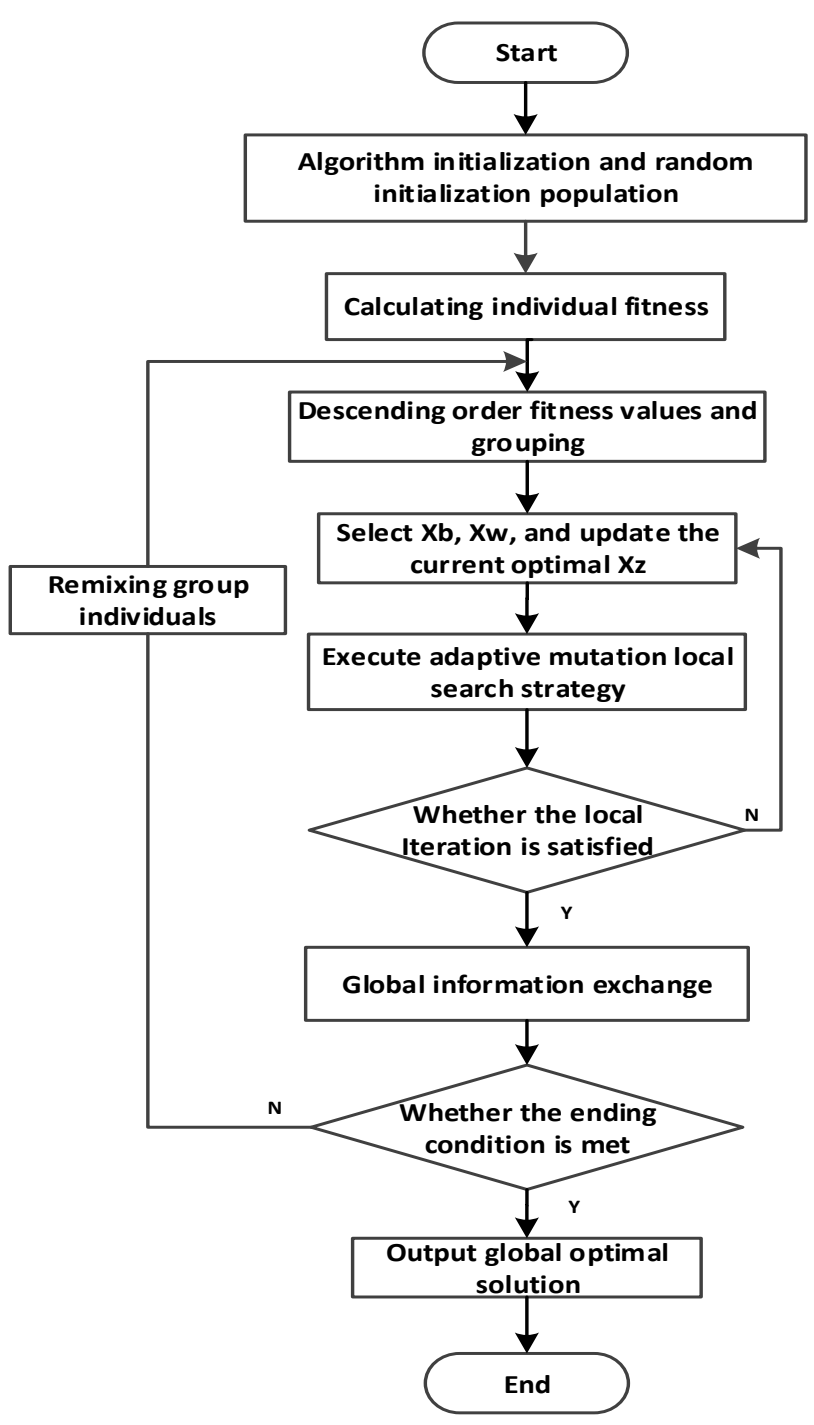

Figure 1. Flow chart of AMSFLA

\section{Location Model of FI in Distribution Network}

The FI location method for distribution networks based on AMSFLA consists of coding, construction of fitness function \& switching function, and adaptive mutation shuffled frog leaping operation. When locating faults, switches (including incoming circuit breakers, sectional switches and loop switches) are used as nodes. Each node is equipped with a FI to collect the fault status of the nodes. Then, the fault signal will be transmitted to the main station through the communication system to evaluate the fault section [21-22].

\subsection{Coding}

This algorithm processes the numeric string of the status information, so it needs to make binary code for the status information of the FI. In the fault location of the distribution network, the fault current goes over the FI at the switch, and section fault statuses are expressed by 1 . The fault currents are not detected by the FI at the switch and section normal statuses are expressed as 0 .

\subsection{Fitness Function}

Establishing a reasonable fitness function is the basis of obtaining an accurate objective function, which affects the accuracy of the algorithm for fault location. When a fault occurs in a distribution network, the FI detects the fault current and sends 
out a discrete signal of 0 or 1 , which is processed by an algorithm to achieve the global optimization of fault location in the distribution network. Therefore, it is necessary to create a fitness function to calculate its fitness. The evaluation function is shown in Equation (8).

$$
F=\sum_{j=1}^{N}\left|I_{j}-I_{j}^{*}\left(B_{N}\right)\right|-\omega \times \sum_{i=1}^{N}|x(i)|
$$

Where $F$ is the fitness and $I_{j}$ is the fault current over-limit signal of the FI at the switch $j$. When the value is 1 , the fault current is detected, and when the value is 0 , the fault current is not detected. $I_{j}^{*}\left(B_{N}\right)$ is the expected status function of the switch $j . N$ is the number of FI installed in the distribution network. $\omega$ takes $\omega=0.8$ as the weight of the total number of fault sections. $x(i)$ is the section i status below fault point, where a value of 1 indicates fault and a value of 0 indicates normal. The entry into $\omega \times \sum_{i=1}^{N}|x(i)|$ avoids misjudgment when FI self-faults.

\subsection{Switching Function}

The switching function reflects the relation between the status of the current over-limit signal at the switch and the fault status of the feeder section. The expected switching status of the distribution network is determined by the line status function. Assuming that the distribution network is divided into $N$ parts by $N$ nodes switches in the line, the status of each switch is determined by its downstream section status. The line status function is shown in Equation (9):

$$
I_{j}^{*}\left(B_{N}\right)=\prod_{i}^{N} x(i)
$$

Where $\prod$ represents logic.

As shown in Figure 2, the construction method of line fault function is introduced by taking a 5 nodes single-source supply radial line as an example. Node 1 is a circuit breaker, nodes 2-5 are sectional switches, and each line is equipped with FI after the node. B1-5 are five sections. Table 1 is the corresponding fault status table for each section. According to the status listed in the table, the switching function can be obtained as follows:

$$
\begin{aligned}
& \text { Figure 2. Structure diagram of radial distribution network with } 5 \text { nodes } \\
& \qquad \begin{array}{l}
I_{1}^{*}\left(B_{N}\right)=x(1) \prod x(2) \prod x(3) \prod x(4) \prod x(5) \\
I_{2}^{*}\left(B_{N}\right)=x(2) \prod x(3) \prod x(4) \prod x(5) \\
\text { Table 1. An example of a table }
\end{array}
\end{aligned}
$$

Table 1. An example of a table
\begin{tabular}{|c|c|c|c|c|c|}
\hline No. $B_{N}$ & $B_{1}$ & $B_{2}$ & $B_{3}$ & $B_{4}$ & $B_{5}$ \\
\hline Section status & $x(1)$ & $x(2)$ & $x(3)$ & $x(4)$ & $x(5)$ \\
\hline
\end{tabular}

Then, the status function of each switch can be obtained by the above method.

After the fault information is confirmed, the adaptive mutation shuffled frog leaping operation can be performed. Then, the location of the fault point can be determined according to the optimal solution obtained by the algorithm.

\section{Conclusions}

This paper takes the typical IEEE 33-bus power distribution system shown in Figure 3 as an example. In the MATLAB 2016a simulation environment, the AMSFLA program is written to calculate the fault information of the distribution network, and the effectiveness of the improved algorithm is verified. 


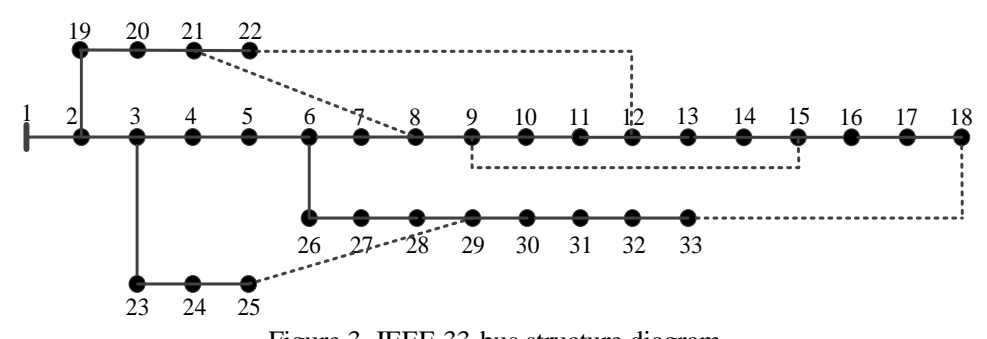

Figure 3. IEEE 33-bus structure diagram

In the simulation, this algorithm has been tested based on single point and multiple point faults. The test results are shown in Table 2.

Table 2. IEEE 33-bus fault test results

\begin{tabular}{|c|c|c|c|c|}
\hline No. & Input fault sequence & $\begin{array}{c}\text { Signal } \\
\text { distortion }\end{array}$ & \multicolumn{2}{|c|}{ Optimum solution } \\
\hline 1 & {$[111110000000000000000000000000000]$} & No & {$[000010000000000000000000000000000]$} & $(5,6)$ \\
\hline 2 & {$[111110000000000000000011000000000]$} & No & {$[000010000000000000000001000000000]$} & $(5,6) /(24,25)$ \\
\hline 3 & {$[111111000000000000111000011111100]$} & No & {$[000001000000000000001000000000100]$} & $(6,7) /(21,22) /(31,32)$ \\
\hline 4 & {$[111010000000000010000000000000000]$} & Yes 4,17 & {$[000010000000000000000000000000000]$} & $(5,6)$ \\
\hline 5 & {$[101110000000010000000011000000000]$} & Yes 2,14 & {$[000010000000000000000001000000000]$} & $(5,6) /(24,25)$ \\
\hline 6 & {$[111011000001000000111000011101100]$} & $\begin{array}{c}\text { Yes } 4,12, \\
29\end{array}$ & {$[000001000000000000001000000000100]$} & $(6,7) /(21,22) /(31,32)$ \\
\hline
\end{tabular}

According to the test results in the above Table 2, when the single point fault and multiple point faults occur in the distribution network, the fault section can be accurately located after the adaptive mutation shuffled frog leaping operation. Comparing No. 1 and No. 4, No. 2 and No. 5, No. 3 and No. 6, when the fault signal is distorted, AMSFLA can still accurately locate the faulty section. The test results show that the improved algorithm for fault information processing can effectively improve the premature problem in the classical SFLA and greatly improve the global optimization of the algorithm.

In order to verify the superiority of AMSFLA applied to fault location, No. 1 is taken as an example to verify the algorithm performance. First, the classical shuffled frog leap algorithm (SFLA), adaptive genetic algorithm (AGA) and adaptive particle swarm optimization algorithm (APSOA) were written in MATLAB. Then, the four algorithms were run 50 times for comparison. The results are shown in Table 3.

Table 3. Algorithm performance test

\begin{tabular}{|c|c|c|c|c|}
\hline Algorithm & $\begin{array}{c}\text { Average } \\
\text { iterations/times }\end{array}$ & $\begin{array}{c}\text { Correct } \\
\text { times/times }\end{array}$ & $\begin{array}{c}\text { Correct } \\
\text { rate/\% }\end{array}$ & $\begin{array}{c}\text { Average time } \\
\text { consuming/ms }\end{array}$ \\
\hline AMSFLA & 32.50 & 48 & 96 & 3.57 \\
\hline SFLA & 55.13 & 45 & 90 & 5.10 \\
\hline AGA & 100.46 & 33 & 66 & 8.20 \\
\hline AMPSO & 63.20 & 39 & 78 & 6.52 \\
\hline
\end{tabular}

The data in Table 3 shows that compared with SFLA, AGA and APSOA, the times of iterations of AMSFLA is significantly reduced, the convergence speed is faster, the accuracy of fault location is higher, and the algorithm performance is superior. AMSFLA can quickly process fault information, find out the fault location in time, and achieve fault location of the distribution network.

\section{Conclusions}

This paper analyzes the shortcomings of the classical SFLA, which is slow in search and easily falls into local optimization. The improved algorithm has introduced the inertia weighting factor and levy mutation operator named AMSFLA, which is applied to fault location of FI in distribution networks, and the algorithm performance is verified by the IEEE 33-bus. The simulation results show that the method has effectively improved the speed of the algorithm and avoids premature convergence. When applied to FI-based fault location, the algorithm can effectively locate single and multiple fault sections. In addition, when the fault information is distorted, it can also accurately distinguish the fault section and provide reliable judgment basis for operation and maintenance staff. 


\section{Acknowledgments}

We thank the support of NSFC with funding numbers U1204506 and 61703144.

\section{References}

1. M. S. Choi, S. J. Lee, and D. S. Lee, "A New Fault Location Algorithm using Direct Circuit Analysis for Distribution Systems," IEEE Transactions on Power Delivery, Vol. 19, No. 1, pp. 35-41, January 2004

2. Z. Z. Guo, Q. X. Xu, and J. J. Hong, "A Novel Fault Section Location Model with Complementarity Constraints and its Optimization Algorithm for Distribution Network," Proceedings of the CSEE, Vol. 36, No. 14, pp. 3742-3751, July 2016

3. Z. Z. Sang, Z. C. Pan, and L. Ding, "The Principle and Application of "S Injection Method" for Faulty Line Selection," Electric Power, Vol. 30, No. 6, pp. 44-45, 1997

4. Z. Qi, B. Z. Liu, and L. Wang, "Application of Wide Area Remnant Current Increment Method of Line Detection in Radial Neutral-Point Resonant Grounded System,” Automation of Electric Power Systems, Vol. 30, No. 3, pp. 84-88, February 2006

5. R. Krishnathevar and E. E. Ngu, "Generalized Impedance-based Fault Location for Distribution Systems," IEEE Transactions on Power Delivery, Vol. 27, No. 1, pp. 449-451, January 2012

6. J. H. Teng, W. H. Huang, and S. W. Luan, "Automatic and Fast Faulted Line-Section Location Method for Distribution Systems based on Fault Indicators," IEEE Transactions on Power Systems, Vol. 29, No. 4, pp. 1653-1662, July 2014

7. M. Farajollahi, M. Fotuhi-Firuzabad, and A. Safdarian, "Deployment of Fault Indicator in Distribution Networks: A MIP-based Approach,” IEEE Transactions on Smart Grid, Vol. 9, No. 3, pp. 2259-2267, May 2018

8. L. Yuan, "Generalized Fault-Location Methods for Overhead Electric Distribution Systems Power Delivery," IEEE Transactions on Industrial Electronics, Vol. 6, No. 1, pp. 53-64, January 2011

9. N. Tong, M. Q. Yu, and X. G. Lin, "Study for Fault Indicator based on the Faulty Phase Current Feature Recognition for the Distribution Network," Transactions of China Electrotechnical Society, Vol. 30, No. 12, pp. 465-471, June 2015

10. J. R. Tang, X. G. Yin, and Z. Zhang, "Survey of Fault Location Technology for Distribution Networks," Electric Power Automation Equipment, Vol. 33, No. 5, pp. 7-13, May 2013

11. J. Zhu, D. L. Lubkeman, and A. A. Girgis, "Automated Fault Location and Diagnosis on Electric Power Distribution Feeders," IEEE Transactions on Power Delivery, Vol. 12, No. 2, pp. 801-809, April 1997

12. J. Liu, J. L. Ni, and Y. Du, "A Unified Matrix Algorithm for Fault Section Detection and Isolation in Distribution System," Automation of Electric Power Systems, Vol. 23, No. 1, pp. 31-33, January 1999

13. N. Mei, D. Y. Shi, and Z. L. Yang, "A Practical Matrix-based Fault Location Algorithm for Complex Distribution Network," Automation of Electric Power Systems, Vol. 31, No. 10, pp. 66-70, May 2007

14. Z. Z. Guo, B. Chen, and C. P. Liu, "Fault Location of Distribution Network based on Genetic Algorithm," Power System Technology, Vol. 31, No. 11, pp. 88-92, June 2007

15. T. Zheng, Y. M. Pan, and K. Y. Guo, "Fault Location of Distribution Network based on Immune Algorithm," Power System Protection and Control, Vol. 42, No. 1, pp. 77-83, January 2014

16. M. M. Eusuff and K. E. Lansey, "Optimization of Water Distribution Network Design using the Shuffled Frog Leaping Algorithm,” Water Resources Planning and Management, Vol. 129, No. 3, pp. 210-225, June 2003

17. T. Niknam, E. A. Farsani, and M. Nayeripour. "A New Tribe Modified Shuffled Frog Leaping Algorithm for Multi-Objective Distribution Feeder Reconfiguration Considering Distributed Generator Units," European Transactions on Electrical Power, Vol. 22, No. 3, pp. 308-333, April 2012

18. A. Rahimi-Vahed and A. H. Mirzaei, "A Hybrid Multi-Objective Shuffled Frog-Leaping Algorithm for a Mixed Model Assembly Line Sequencing Problem," Computers and Industrial Engineering, Vol. 53, No. 4, pp. 642-666, November 2007

19. X. D. Zhang, Y. F. Zhang, and Y. H. Shi, "Power Control Algorithm in Cognitive Radio System based on Modified Shuffled Frog Leaping Algorithm," International Journal of Electronics and Communications, Vol. 66, No. 6, pp. 448-454, June 2012

20. E. Elbeltagi, T. Hezagy, and D. Grierson, "Comparison among Five Evolutionary-based Optimization Algorithms," Advanced Engineering Informatics, Vol. 19, No. 1, pp. 43-53, January 2005

21. T. S. Yan, D. W. Cui, and Y. Q. Tao, "Fault Location for Distribution Network by Improved Genetic Algorithm," High Voltage Engineering, Vol. 35, No. 2, pp. 255-259, February 2009

22. Z. Z. Guo and J. K. Wu, "Electromagnetism-Like Mechanism based Fault Section Diagnosis for Distribution Network," Proceedings of the CSEE, Vol. 30, No. 13, pp. 34-40, May 2010

All authors are affiliated with the Engineering and Automation School at Henan Polytechnic University.

Yanzhou Sun is a Professor with a Doctoral degree from Xi'an Jiaotong University. His research focuses on power supply technology including high voltage engineering.

Han Wu and Yawei Zhu are Master's students.

Yanfang Wei is an Associate Professor with a Doctoral degree from Hohai University. He is working on power systems including analysis, control, transmission, and distribution.

Tieying Zhao is a Lecturer with a Doctoral degree from China University of Mining and Technology. 